\title{
Detonation initiation developing from the Richtmyer-Meshkov instability
}

\author{
H. H. Teng $\cdot$ Z. L. Jiang $\cdot$ Z. M. Hu
}

Received: 29 August 2006 / Revised: I February 2007 / Accepted: 6 March 2007 / Published online: 24 July 2007

(c) Springer-Verlag 2007

\begin{abstract}
Detonation initiation resulting from the Richtmyer-Meshkov instability is investigated numerically in the configuration of the shock/spark-induced-deflagration interaction in a combustive gas mixture. Two-dimensional multi-species Navier-Stokes equations implemented with the detailed chemical reaction model are solved with the dispersion-controlled dissipative scheme. Numerical results show that the spark can create a blast wave and ignite deflagrations. Then, the deflagration waves are enhanced due to the Richtmyer-Meshkov instability, which provides detonation initiations with local environment conditions. By examining the deflagration fronts, two kinds of the initiation mechanisms are identified. One is referred to as the deflagration front acceleration with the help of the weak shock wave, occurring on the convex surfaces, and the other is the hot spot explosion deriving from the deflagration front focusing, occurring on the concave surfaces.
\end{abstract}

Keywords Hot spot - Deflagration front acceleration . Detonation injtiation - Richtmyer-Meshkov instability

\section{Introduction}

Detonation initiation is one of the most fundamental and difficult problems in the detonation physics. The direct initiation and the initiation via a deflagration to detonation transition (DDT) are two primary kinds of the detonation initiation. The DDT process is more commonly observed in practice,

The project supported by the National Natural Science Foundation of China (90205027 and 10632090$)$.

H. H. Teng $\cdot$ Z. L. Jiang $(\bowtie) \cdot$ Z. M. Hu

LHD, Institute of Mechanics. CAS, Beijing 100080, China

e-mail: zljiang@imech.ac.cn but very difficult to describe quantitatively. This is because the DDT usually involves shock interactions, turbulent mixing, chemical reactions, flow and chemistry instability, which results in the DDT process being very sensitive to the initial and boundary conditions. Therefore, it is identitied as a highly nonlinear and multi-scale flow phenomenon. The investigation on detonation initiation has not only a theoretical meaning, but also important application backgrounds in some fields, such as pulsed detonation engines and the explosion hazard prevention in chemical plants.

Urtiew and Oppenheim [1] first observed the detonation initiation deriving from hot spots in the DDT. Thereafter. many experiments were carried out by using different ignition methods and the hot spots were observed universally [2]. Oran and Khokhlov et al. $[3,4]$ numerically simulated the interaction between shock waves and deflagrations. in which it was observed that the hot spots developed from Richtmyer-Meshkov instability. Due to the universal appearing of hot spots in the detonation initiation, its evolution process was investigated widely [5-7]. However its forming process and detail development mechanism are still unclear because the hot spot usually appears instantaneously in highspeed turbulent deflagration and the position in which it may be observed is random case by case. Therefore, the hot spot forming process and its decisive factors are worthy of more attention to understand the physical essence of the detonation initiation.

Detonation initiation deriving from shock waves is a typical research topic. The shock wave can compress gases and increase its temperature to an auto-ignition level, and detonation initiations may take place [8]. Because very strong shock waves are necessary to achieve the detonation initiation quickly, some investigations on the detonation initiation are carried out on the basis of the shock wave reflections [9-11] or shock wave focusing [12]. For detonation initiations, using 
a spark for detonation initiation is also a popular way in engineering applications, but the spark-induced DDT is usually a lengthy process since the ordinary spark plugs cannot release enough energy for a direct initiation. However, it must be easier to initiate the detonation quickly if combining the above-mentioned two methods. In the case, the Richtmyer-Meshkov instability resulting from the incident shock/spark-induced-deflagration interaction may lead to the combustion enhancement and further achieve a faster detonation initiation. The objective of this paper is to simulate the incident shock/spark-induced-deflagration interaction and the Richtmyer-Meshkov instability to demonstrate the role of the interface instability in the detonation initiation. The physical model under the consideration and the applied numerical methods will be introduced in Sect. 2 . The evolution of the Richtmyer-Meshkov instability and the relevant detonation initiation will be described in Sect. 3, including observations on the deflagration acceleration, the hot spot development, local explosions and detonation bubbles. The conclusions reached will be presented in Sect. 3.2.

\section{Physical model and numerical methods}

The sketch of the incident shock/spark-induced-deflagration interaction is shown in Fig. 1. The computational domain is a two-dimensional and filled with premixed combustive gas mixtures, a spark is released at the centerline and drives out a blast wave followed by a flame interface. And then, the incident shock interacts with the blast wave and later with the flame interface separating the burnt products from the reactants. The interface will go into an unstable state due to the Richtmyer-Meshkov instability mechanism, and hot spots and flame accelerations may occur on the flame interface, from which the detonation initiation is achieved finally. In the simulation the spark locates on the plane of symmetry initially, and the incident shock propagating from left to right is set near the left boundary, as shown in Fig. 1. The spark is not strong enough to initiate a detonation but is sufficiently powerful to ignite a deflagration. Because this initiation process is almost instantaneous, the turbulence effect can be negligible. Therefore, the governing equations are

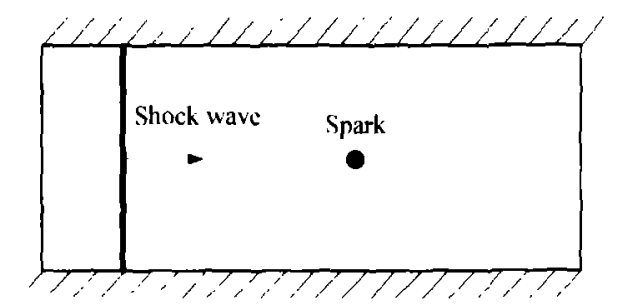

Fig. 1 Sketch of the incident shock/spark-induced-deflagration interaction simplified as two-dimensional multi-species Navier-Stokes equations which can be written as follows:

$$
\frac{\partial U}{\partial t}+\frac{\partial F}{\partial x}+\frac{\partial G}{\partial y}+S_{\iota}=\frac{\partial F_{v}}{\partial x}+\frac{\partial G_{v}}{\partial y} .
$$

where

$$
\begin{aligned}
U & =\left[\begin{array}{lllllll}
\rho_{1} & \rho_{2} & \cdots & \rho_{n} & \rho u & \rho v & e
\end{array}\right]^{\mathrm{T}}, \\
F & =\left[\begin{array}{lllllll}
\rho_{1} u & \rho_{2} u & \cdots & \rho_{n} u & \rho u^{2}+p & \rho u v & (e+p) u
\end{array}\right]^{\mathrm{T}}, \\
G & =\left[\begin{array}{lllllll}
\rho_{1} v & \rho_{2} v & \cdots & \rho_{n} v & \rho u v & \rho v^{2}+p & (e+p) v
\end{array}\right]^{\mathrm{T}}, \\
S_{c^{\prime}} & =\left[\begin{array}{lllllll}
\dot{\omega}_{1} & \dot{\omega}_{2} & \cdots & \dot{\omega}_{n} & 0 & 0 & 0
\end{array}\right]^{\mathrm{T},} \\
F_{v^{\prime}} & =\left[\begin{array}{llllllll}
0 & 0 & \cdots & 0 & \tau_{x x p} & \tau_{x y} & u \tau_{x x p}+v \tau_{x y}-q_{x}
\end{array}\right]^{\mathrm{T}}, \\
G_{l^{\prime}} & =\left[\begin{array}{llllllll}
0 & 0 & \cdots & 0 & \tau_{x y} & \tau_{y y p} & u \tau_{y y}+v \tau_{y y p}-q_{y}
\end{array}\right]^{\mathrm{T}},
\end{aligned}
$$

in which $\rho_{i}(i=1, \ldots n)$ is the $i$ th specie density and the total density $\rho=\sum_{i=1}^{n} \rho_{i} ; u$ and $v$ are the velocity component in the $x$ - and $y$-direction. respectively; $e$ is the total specific energy and $e=\rho h-p+1 / 2 \times \rho\left(u^{2}+v^{2}\right)$, where the specific enthalpy of the gas mixture can be written as $h=\sum_{i=1}^{n} \rho_{i} h_{l} / \rho$ and the $i$ th specie specific enthalpy $h_{i}$ can be calculated with curve fitting [13]; $p$ stands for gas pressure and can be written as $p=\sum_{i=1}^{\prime \prime} \rho_{i} R_{i} T$, where $R_{i}$ is the $i$ th specie gas constant and $T$ is the gas temperature; $\dot{\omega}_{i}$ is the $i$ th specie specific mass production rate, and may be expressed in different ways in different chemical reaction models. The viscous terms can be easily found out in some text books, and therefore will be not presented in this paper.

Dispersion-controlled dissipative scheme [14], which is proved to be efficient and robust in the shock-capturing simulation, is adopted to discrete the governing equations. The chemical reaction model is very important in detonation initiation simulations and over-simplified physical models may result in meaningless results [15]. Therefore, the detailed chemical reaction model of the hydrogen/air mixture is applied with the CHEMKIN package [16], which includes 8 species $\left(\mathrm{H}_{2}, \mathrm{O}_{2}, \mathrm{O}, \mathrm{H}, \mathrm{OH}, \mathrm{HO}_{2}, \mathrm{H}_{2} \mathrm{O}_{2}, \mathrm{H}_{2} \mathrm{O}\right)$ and 20 reactions. The stiff problem deriving from chemical reactions is solved by DVODE package [17]. Initially the computational domain is filled with the premixed reactive gas mixture at the condition of $1.0 \mathrm{~atm}, 298.15 \mathrm{~K}, 2 \mathrm{H}_{2}+\mathrm{O}_{2}$. The periodical boundary is specified both on the upper and low boundaries to simulate multi-sparks initiation. The inflow and outflow boundaries are interpolated under the assumption of the zero first-order derivatives of all flow parameters. The computational domain is $2 \mathrm{~mm} \times 10 \mathrm{~mm}$. The grid is the orthogonal Cartesian mesh with the mesh scale of $0.01 \mathrm{~mm}$ in both directions. The incident shock of the Mach number 1.6 is set at the position of $x=0.1 \mathrm{~mm}$ and the spark is posited at the $x=1.0 \mathrm{~mm}$. The spark is supposed to be a circular zone with a six-grids diameter, and thermodynamic parameters within the zone are homogeneous and calculated according to the 
constant volume combustion assumption of the presentlyused gas mixture.

\section{Numerical results and discussion}

\subsection{Deflagration development}

Time sequential pressure (upper) and temperature (lower) distributions during the deflagration developing process are shown in Fig. 2. A circular blast wave is generated after the park releasing, travels outward, and becomes weaker due to the successive expansion waves. Behind the blast wave there is a high-temperature reactive zone separating reactants from products. The incident shock impacts on the blast wave and the reactive zone develops, as shown in Fig. 2a. The incident shock interacts with the reaction zone in Fig. $2 b$, which results in the curved incident shock since the locally-developed nonuniform velocity field induced by the blast wave, and the curved reactive zone due to shock acceleration. Once the incident shock passes over, the reactive zone expands quickly as shown in Fig. 2c. This implies that the intense exothermic reactions occur due to the higher shock-induced temperature. Series compression waves can be identified near the reactive zone both upstream and downstream. The upstream one derives from the shock/flame interaction and the downstream one derives from the heat-released acceleration that is getting more intensive as shown in Fig. $2 \mathrm{~d}$. This result demonstrates the high-speed deflagration development due to the positive feedback loop of the reaction/compression-waves interaction, that is, the deflagration acceleration induces the compression waves that compress the gas mixture in front of the reactive zone, and in return enhance the deflagration. As the incident shock and the reactive zone travel downstream, the chemical reactions are getting more and more intensive, and the downstream compression waves becomes stronger, but the distance between the incident shock and the reactive zone becomes longer and longer, which demonstrates that there is only the deflagration wave in the present stage.

Pressure and temperature profiles on the plane of symmetry during the deflagration development are shown in a time sequence in Fig. 3. The four profiles are corresponding to the flow fields shown in Fig. 2. The upstream-traveling blast wave has a higher pressure peak due to the incident shock compression, as shown with the first profile in the left of Fig. 3a, while the downstream-traveling part is weaker and dissipated gradually. The second pressure jump is induced by the incident shock, propagates faster and catches up with the blast wave in the last stage. From Fig. $3 b$, it can be found that the peak temperature is low in the reactive zone before the incident shock arrives, then becomes higher and higher after the shock interaction, and finally reaches to about $3,000 \mathrm{~K}$. The temperature increase of the reactive zone benefits from the elevated temperature of the shocked flow and the shock-induced chemical reaction acceleration, and the later phenomenon has the greater contribution. In the temperature-
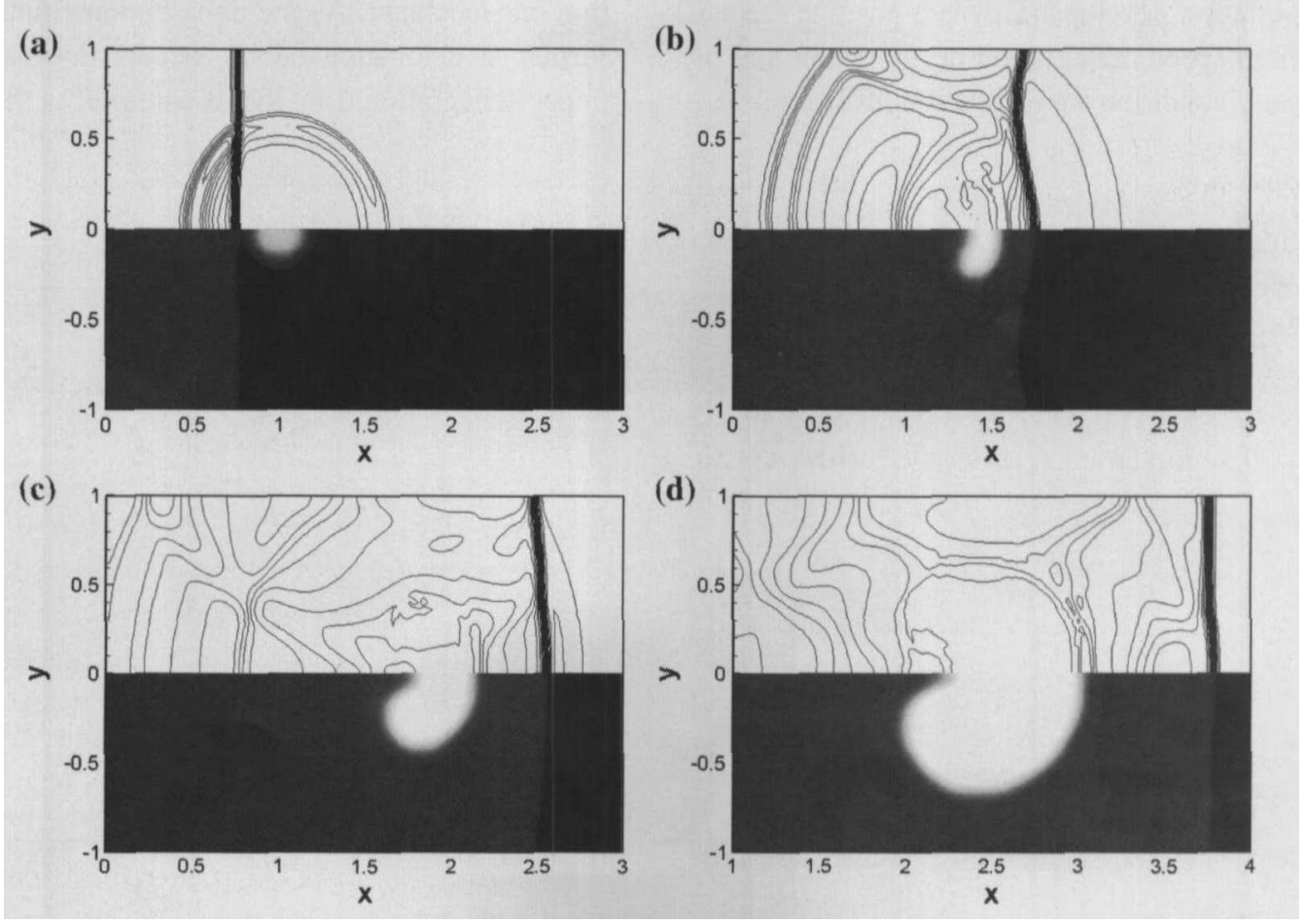

Fig. 2 Time sequential pressure (upper) and temperature (lower) distributions in the deflagration developing process after the spark releasing 
Fig. 3 Pressure and temperature profiles along the plane of symmetry during the deflagration developing process (a)

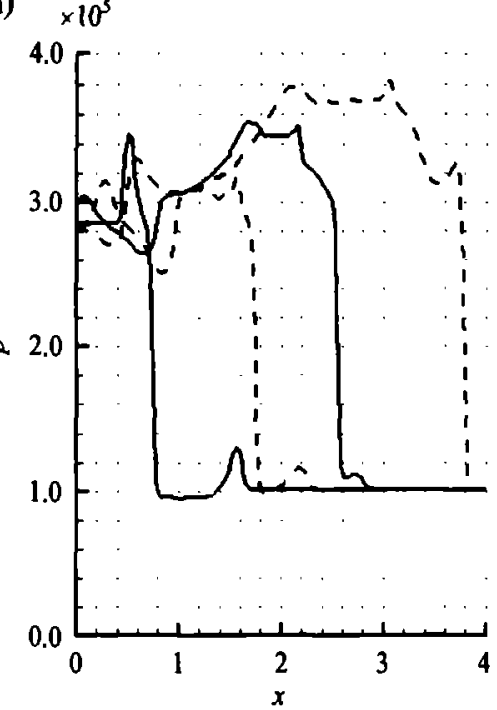

(b)

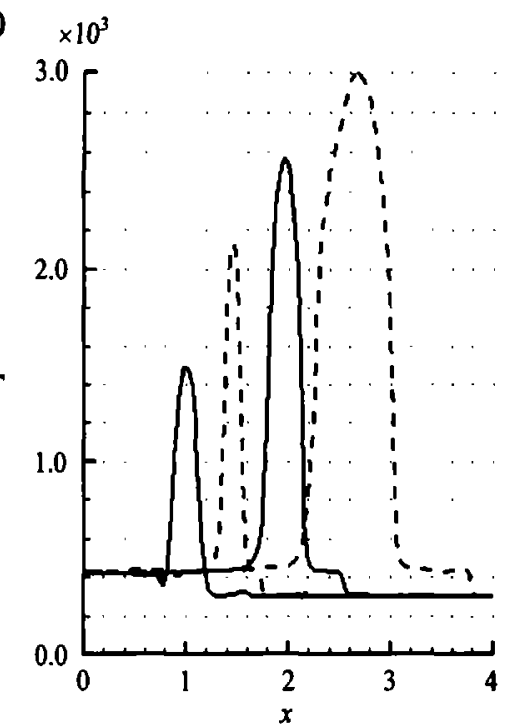

rising process, the pressure around the reactive zone also rises a little due to compression waves generation, as shown in Fig. 3a, but there are no obvious shock waves to be identified. Figure $3 \mathrm{~b}$ shows two temperature jumps, the one is lower and generated due to incident compression, and the other one is much higher and induced by flame acceleration. The interval between the two jumps is getting wider and wider, which indicates that no direct detonation initiation occurs.

From above discussions, it is concluded that the spark ignites a deflagration, and the incident shock wave makes the deflagration more intensive. The intensive deflagration induces compression waves that elevate the flow temperature in front of the deflagration front, from which the more intensive deflagration develops. This is a positive feedback loop for the high speed deflagration development and provides the primary condition for detonation initiations.

\subsection{Detonation initiation from Richtmyer-Meshkov instability}

It is shown in Fig. 4 that the deflagration front goes unstable after the incident shock passes away, and the detonation initiation takes place due to Richtmyer-Meshkov instability. It can be observed that the deflagration front developed well into a rather irregular pattern and occupied the whole cross section. There are no obvious shock-induced pressure jumps in front of the deflagration front, as shown in Fig. 4a, but some compression waves are clearly observable in the front of either the convex front upstream or the concave front downstream. These compression waves developed from deflagration enhancement. As the deflagration fronts are enhanced further, a detonation bubble occurs downstream near the upper deflagration front that is concave, as shown in Fig. 4b.
Fig. 4 Time sequence of pressure (upper) and temperature (lower) distributions in the detonation initiation process
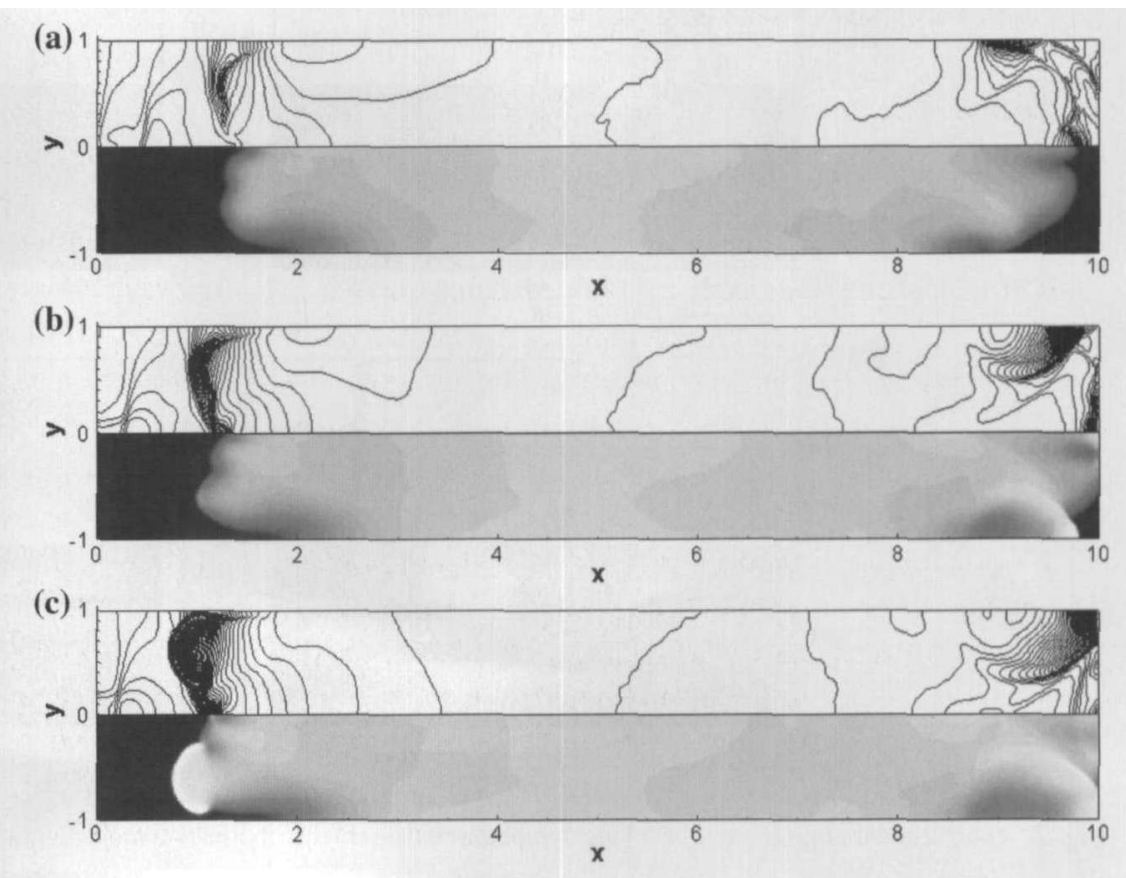
Fig. 5 Time sequence of pressure (upper) and temperature (lower)

distributions of the enlarged local flow field downstream during the first detonation bubble development
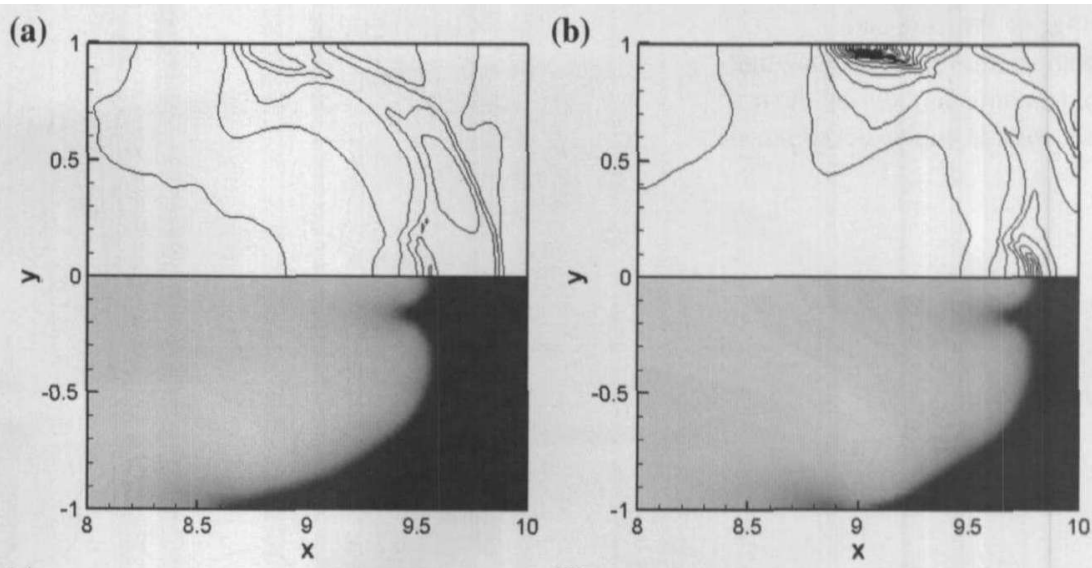

(c)

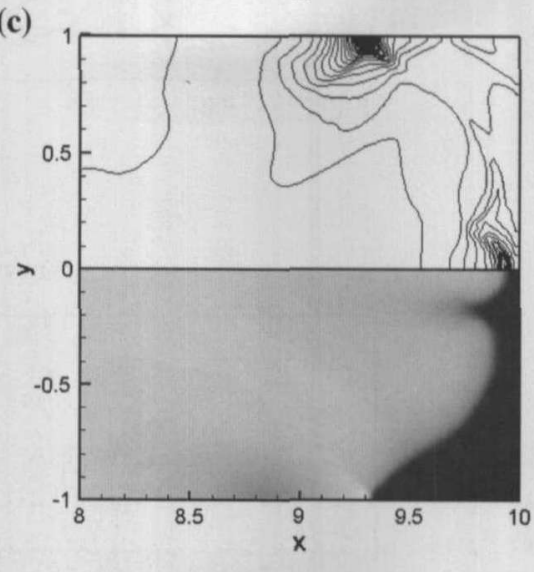

(d)

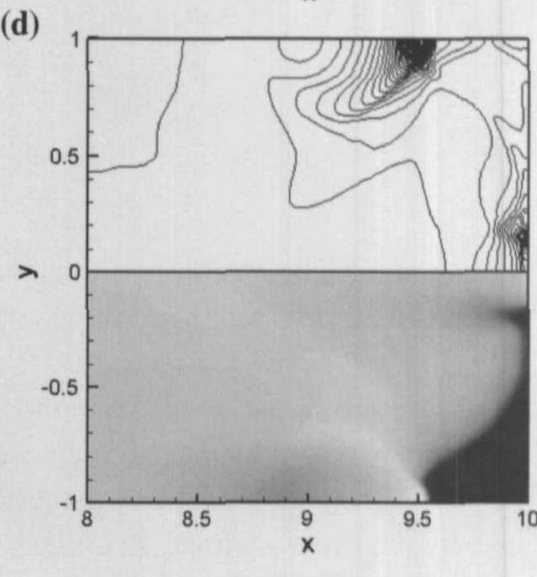

Meanwhile, the upstream compression waves become stronger and a relevant small high temperature zone can be observable near the convex deflagration front. And then the second detonation bubble develops and expands quickly, as shown in Fig. 4c. The second bubble is generated at the tip of the convex deflagration front, and is different from the first one resulting from the concave front focusing. Two detonation bubbles develop quickly into two detonation waves that travel upstream and downstream, separately.

\subsection{Discussion on the initiation mechanism}

The detonation initiation from the first detonation bubble in the flow field downstream is shown in Fig. 5. The deflagration enhancement induces a wrinkled flame surface in front of which a series of compression waves are observable, as shown in Fig. 5a. The compression waves become more intensive and a concave flame front forms at the upper boundary, as shown in Fig. 5b. This concave front focuses and generates a higher pressure zone, as shown in Fig. 5c. The coupling of the compression waves and the high temperature zone indicates the hot spot generation from which a detonation bubble forms, as shown in Fig. 5d. This initiation derives from the hot spot explosion, and is similar to many previous experimental results published. The hot spot occurs at the concave deflagration front and is generated due to the deflagration front focusing.
Pressure and temperature profiles along the upper boundary during the first detonation bubble development are shown in Fig. 6. It is observable that the pressure rises quickly to its peak value and then falls gradually to a constant value. The peak pressure benefits from the concave deflagration front focusing, and the finally-reached constant value indicates the stable detonation, where both the pressure and temperature peaks appear on the detonation wave front. The pressure peak rises to a maximum value and then falls, while the temperature peak rises to about $4,000 \mathrm{~K}$ and keeps nearly constant. As well known, this is a typical process of the overdriven detonation initiation by the hot spot explosion.

The process of the second detonation bubble generation upstream is shown in Fig. 7. The deflagration front and its relevant compression waves can be observed in Fig. 7a. The front is convex, so both the deflagration and the compression waves are expected to become weaker and weaker due to the wave diverging effect. However, the compression waves become stronger and stronger as shown in Figs. 7b, c. And finally the arc-shaped detonation wavelet forms, as shown in Fig. 7d. In this process, the continuously-enhanced deflagration can generate a series of strong compression waves, which can over-compensate the wave diverging effects and are accumulated in the front of the deflagration front. The accumulated compression waves result in the temperature peak at the tip of the convex deflagration front, and more intensive chemical reactions are triggered. This is a positive 
Fig. 6 Pressure and temperature profiles along the upper boundary during the first detonation bubble development

Fig. 7 Time sequence of pressure (upper) and temperature (lower) distributions of the enlarged local flow field upstream during the second detonation bubble development
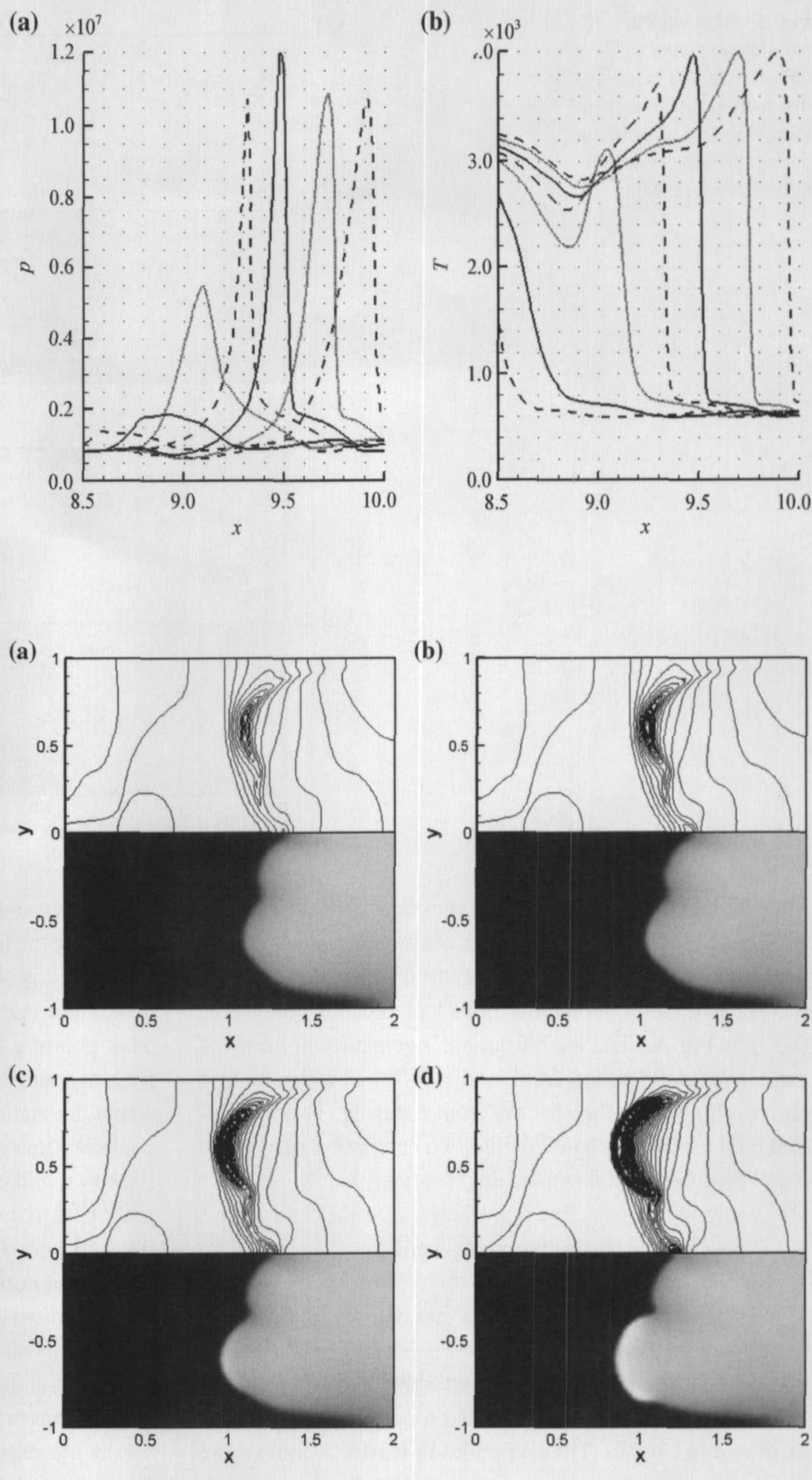

feed-back loop as mentioned before, which is vital in the detonation initiation from a convex deflagration front.

The pressure and temperature profiles along the line $x=0.06$ during the second detonation bubble development upstream are shown in Fig. 8. As the deflagration front moves upstream the pressure peak increases gradually and reaches to an almost constant value with small fluctuations in the last stage. No obvious over-shot pressure is observable as the one in the hot spot case and the detonation initiation is a deflagration accelerating process. Meanwhile the temperature peak does in the same way as the pressure peak does, reaches its maximum value gradually and keeps nearly constant later. 
Fig. 8 Pressure and temperature profiles along the line $x=0.06$ during the second detonation bubble development (a)

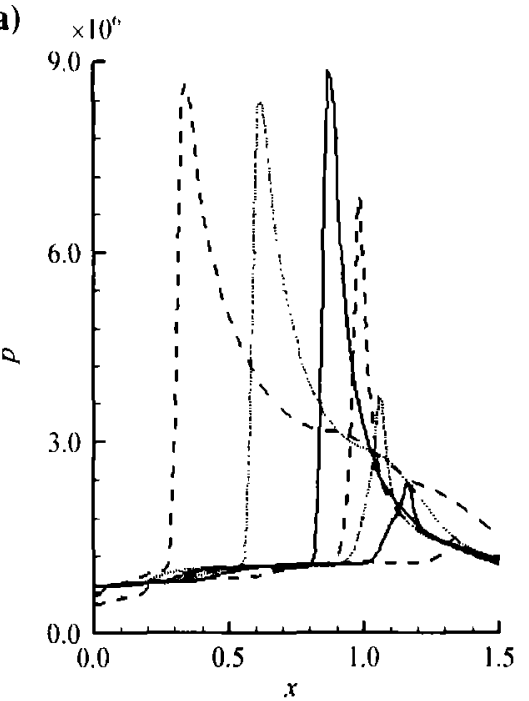

(b)

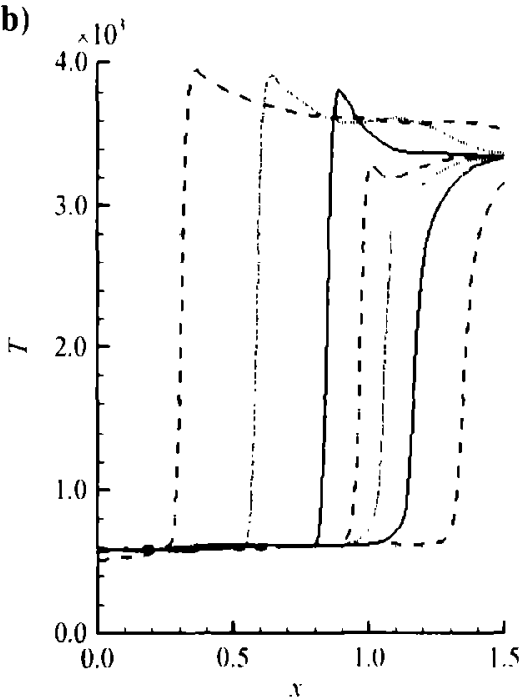

Without the overdriven detonation, the temperature behind the shock is almost constant, which is different essentially from the falling profiles shown in Fig. 6 b.

\section{Concluding remarks}

Detonation initiations resulting from the RichtmyerMeshkov instability are examined in the case of the shock/ spark-induced-deflagration interaction. Two initiation processes are identified and their underlying mechanisms are discussed. One of them is referred to as the deflagration acceleration with the help of the compression waves occurring on the convex surfaces. In this mode, the detonation is reached gradually, and no obvious over-shot pressure and temperature are observable. The other one is called as the hot spot explosion deriving from the concave deflagration front focusing. In the mode, an over-driven detonation is generated first from strong explosion, and then decays to the steady detonation. The positive feedback loop of deflagration/compression-wave interaction is the fundamental mechanism for the two detonation initiations resulting from the Richtmyer-Meshkov instability and is more important in the deflagration acceleration process.

\section{References}

1. Urtiew, P., Oppenheim, A.K.: Experimental observations of the transition to detonation in an exploding gas. Proc. R. Soc. Lond. A 295, 13-18 (1966)

2. Lee, J.H.S., Moen, I.O.: The mechanism of transition from deflagration to detonation in vapor cloud explosions. Prog. Energy Combust. Sci. 6, 359-389 (1980)
3. Oran, E.S., Khokhlov, A.M.: Deflagrations, hot spots, and the transition to detonation. Phil. Trans. R. Soc. Lond. A 357. 35393551 (1999)

4. Khokhlov, A.M., Oran, E.S., Thomas, G.O.: Numerical simulition of deflagration-to-detonation transition: the role of shockflame interactions in turbulent flames. Combust. Flame 117. 323-339 ( 1999 )

5. Lee, J.H.S.: Detonation waves in gaseous explosives. In: Ben-Dor G, Igra O, Elperin T (eds) Handbook of Shock Waves, vol. 3. pp. 309-415. Academic. San Diego (2001)

6. Thomas, G.O., Jones, A.: Some observations of the jet initiation of detonation. Combust. Flame 120, 392-398 (20)(0)

7. Khokhlov, A.M., Oran, E.S.: Numerical simulation of detoniltion initiation in a flame brush: the role of hot spots. Combust. Flame 119, 400-416 (1999)

8. Brown, C.J., Thomas, G.O.: Experimental studies of shockinduced ignition and transition to detonation in ethylene and propane mixtures. Combust. Flame 117, 861-870) (1999)

9. Kaplan, C.R., Oran, E.S.: Mechanism of ignition and detonalion formation in propane-air mixtures. Combust. Sci. Tech. 80. 185205 (1981)

10. Chan, C.K.: Collision of a shock wave with obstacles in a combustible mixture. Combust. Flame 100, 341-348 (1945)

11. Brown, C.J.. Thomas, G.O.: Experimental studies of ignition and transition to detonation induced by the reflection and diffraction of shock waves. Shock Waves 10. 23-32 (2000)

12. Gelfand, B.E., Khomik, S.V., Bartenev, A.M., et al.: Detonation and deflagration initiation at the focusing of shock waves in combustible gaseous mixture. Shock Waves 10, 197-2()4 (2(K))

13. McBride, B.J., Zehe, M.J., Gordon, S.: NASA glenn coefficients for calculating thermodynamic properties of individual species. NASA TP 2002-211556 (2002)

14. Jiang, Z: On dispersion-controlled principles for non-oscillatory shock-capturing schemes. Acta Mech. Sin. 20. 1-15(2(1)-4)

15. Lee, J.H.S., Higgins, A.J.: Comments on criteria for direct initiation of detonation. Phil. Trans. R. Soc. Lond. A 357, 3503 3521 (1999)

16. Kee, R.J., Rupley, F.M., Meeks, E., et al.: Chemkin-III: a fortran chemical kinetics package for the analysis of gas-phalse chemical and plasma kinetics. UC-405, SAND96-8216, May 1996, Sandia National Laboratories, Livermore, CA

17. Brown, P.N.. Byrne, G.D., et al.: VODE, a variable-coefficient ode solver. SIAM J. Sci. Stat. Comput. 10, 10.38-1051 (1989) 